\title{
Do we need a retinal pigment epithelium (or choroid) for the maintenance of retinal apposition?
}

\author{
WALLACE S FOULDS \\ From the Tennent Institute of Ophthalmology, University of Glasgow
}

SUMMARY The relative rarity of retinal detachment following extensive surgical resection of choroid and retinal pigment epithelium for choroidal malignant melanoma is discussed in relation to the factors maintaining retinal apposition under these circumstances.

Many structural and dynamic factors have been suggested as being of importance in the maintenance of retinal apposition with its bed. ${ }^{2}$ These include interdigitation of retinal receptor cell outer segments with the apical processes of the retinal pigment epithelium (RPE), the presence of the interphotoreceptor matrix between the outer retina and the RPE, ${ }^{3}$ and active transport of molecules from the potential subretinal space to the choroid by cells of the RPE. ${ }^{4}$ All these suggested mechanisms presuppose either a structural or functional role for the RPE in the maintenance of retinal apposition.

Experience of local resection of choroidal melanoma has indicated that surgical removal of extensive areas of choroid and RPE does not commonly lead to retinal detachment, and the significance of this finding is discussed in this paper.

\section{Materials and methods}

The technique used by the author for the local surgical resection of choroidal melanomas has been previously described ${ }^{5-7}$ and has now been applied in more than 100 cases. The technique involves the surgical removal of affected choroid and related RPE together with a half thickness deep lamella of sclera adjacent to the choroid containing the tumour. The overlying retina is separated from the tumour and in most instances remains intact. The wall of the eye is reconstituted by the outer lamella of sclera, which has been temporarily raised as a flap to allow access to the affected choroid and related deep scleral lamella.

Whether there is a preoperative retinal detachment or not-and the presence of an exudative Correspondence to Professor W S Foulds, Tennent Institute of Ophthalmology, Western Infirmary, Glasgow G11 6NT. detachment is of course common in cases of choroidal melanoma-it is a constant finding that in the immediate postoperative period the retina is flat and in contact with the scleral bed (Figs. 1 and 2). Flattening of the retina does not appear to be dependent on the area of resection and occurs even where up to one-third of the choroid and RPE has been removed (Fig. 3).

Occasionally a tumour is adherent to the overlying retina, and in these cases a full thickness breach of the retina may occur during surgical removal of the tumour. In spite of this, retinal flattening on to the scleral bed is still the rule provided the retinal hole is over the resected area.

Sometimes late retinal detachment has been seen where vitreous haemorrhage has led to the development of preretinal fibrosis and traction (in the absence of a retinal break) or very occasionally where

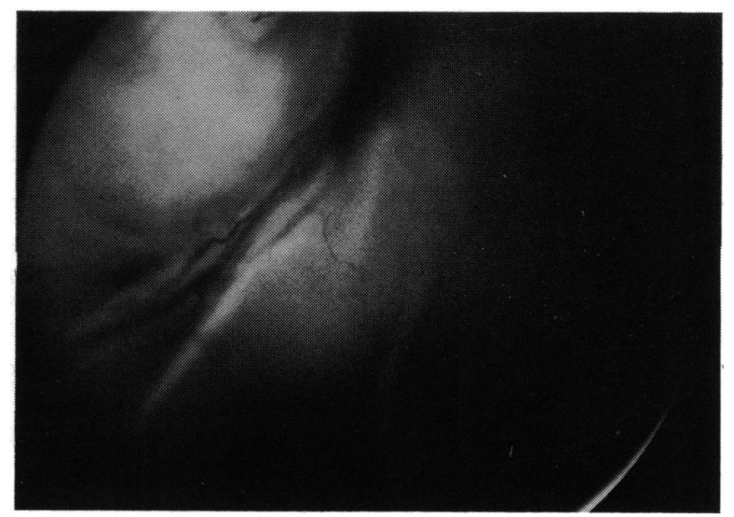

Fig. 1 A large choroidal melanoma complicated by extensive retinal detachment. The tumour was treated by local surgical excision. 


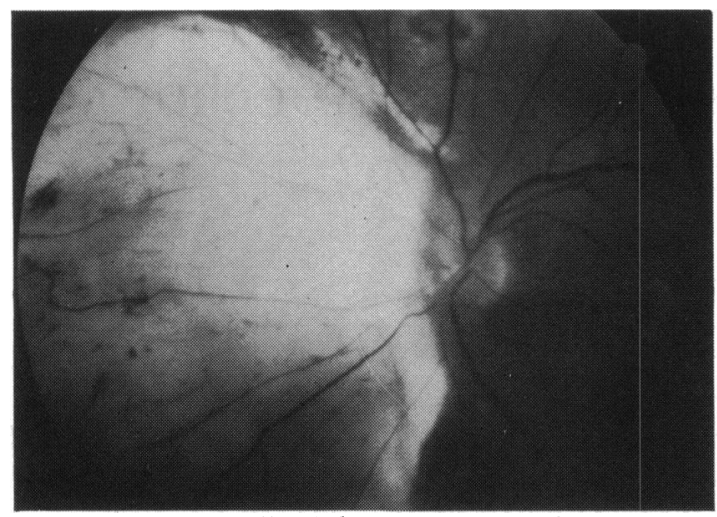

Fig. 2 Same eye as Fig. 1 showing postoperative appearance. The retina is now flat and remains in contact with bare sclera in the surgical coloboma.

a full thickness retinal tear has been situated at the edge of the resected area rather than centrally. In these cases the previously flat retina has separated from the underlying scleral bed without evidence of acquired adhesion between the retina and bare sclera.

\section{Results}

Of the first 90 cases of local resection for choroidal melanoma there was subsequent retinal detachment in 14 cases. In nine instances the retinal detachment was the result of vitreous organisation and traction

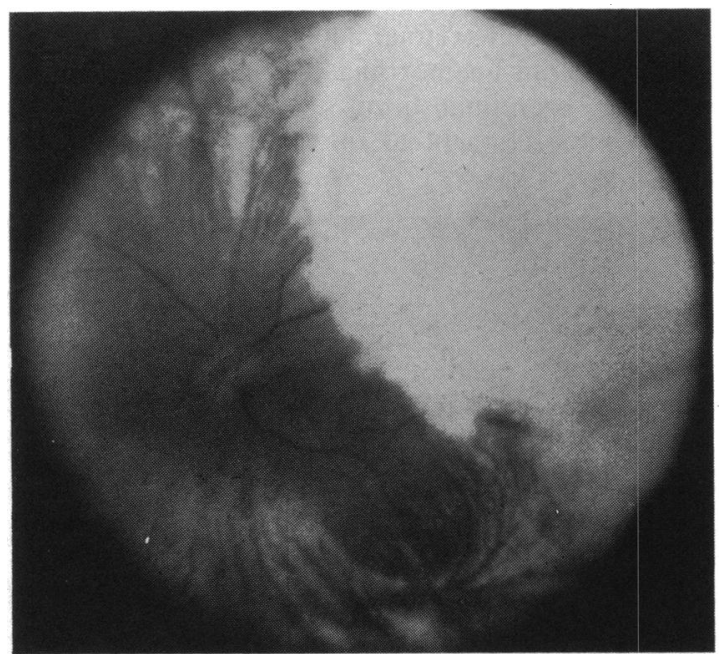

Fig. 3 Wide-angled fundus photograph to show postoperative appearance after local surgical resection of a very large choroidal tumour. The surgical coloboma involves one-third of the choroid and pigment epithelium but the overlying retina remains flat and in contact with bare sclera. and unassociated with a detected retinal break. In two of these cases retinal reattachment followed closed pars plana vitrectomy and fluid gas exchange. In five cases the retinal detachment was a complication of retinal hole formation close to the resected area. In four of the five cases retinal reattachment followed conventional detachment surgery.

\section{Discussion}

The results in cases of local choroidectomy show that even a previously detached retina will flatten on to bare sclera after surgical removal of choroid and RPE and in most cases remain in contact with the scleral bed, even though no actual adhesion may form between retina and sclera. It is interesting to speculate on the forces involved in this retinal apposition with the sclera.

It is known that in the normal animal eye small molecules injected into the vitreous (xenon, ${ }^{x}$ tritiated water ${ }^{y}$ ) largely leave by passing across the retina and RPE to reach the choroid. Each of these structures offers resistance to the passage of small molecules, and we and others have determined the flow conductivity of retina, ${ }^{10}$ combined retina, RPE, and choroid, ${ }^{11}$ and sclera. ${ }^{12}$ Not unexpectedly the sclera has a higher resistance per unit area to the passage of water than does either retina or combined retina, RPE, and choroid. ${ }^{\prime \prime}$

We have shown that there is a small movement of water and other tracers from the vitreous out through the sclera, but in the intact eye this is at most only $2.4 \%$ of total tracer injected into the vitreous. ${ }^{13}$

The rate of molecular movement from the subretinal space to the choroid across the RPE is significantly increased following metabolic damage to the RPE by intravenously injected sodium iodate, ${ }^{14}$ suggesting that intact RPE acts as a barrier in relation to the outward movement of molecules from the subretinal space. Any such barrier will undoubtedly be destroyed when the RPE is surgically removed.

As a working hypothesis it is suggested that the reduction in scleral thickness, which is an inherent component of the technique used for the surgical resection of malignant melanoma, may decrease scleral resistance and increase transscleral flow. The removal of choroid and RPE will in addition remove any resistance in these tissues to the passage of fluid from the potential subretinal space to the sclera. Under these circumstances the relative flow conductivities of the retina and of the surgically thin sclera would be important. If fluid escaped transsclerally at a rate which was potentially greater than its complementary movement across the retina, then a small pressure difference on either side of the retina would be generated and might be sufficient to main- 
tain the retina in apposition with the sclera. It seems unlikely, especially in larger resections, that the intact RPE at the edge of the surgical coloboma could empty the subretinal space laterally to maintain the required apposition.

That the resulting apposition of retina with the sclera is maintained by functional rather than structural forces is suggested by the occasional late occurrence of retinal detachment as a complication of preretinal traction or a retinal break not situated immediately over the resected area. In those cases where this has occurred the retina appears to have become detached from the scleral bed without apparently having established any structural union with it.

The fact that a full thickness retinal break when situated over the resected area is not as a rule complicated by retinal detachment postoperatively is surprising. It is possible that in some cases tamponade of the break by formed cortical vitreous may maintain a sufficient resistance to the passage of water from the vitreous cavity to the potential subretinal space. ${ }^{15} \mathrm{An}$ alternative explanation might relate to the mathematics of water movement through the retinal break relative to water movement through the sclera. A similar mechanism has been postulated and subjected to mathematical investigation in the case of retinal flattening after episcleral plombage where a retinal hole is not in contact with the indentation at the end of the surgical procedure. ${ }^{16}$ In the case of local resection of choroidal tumours, however, it has been the author's experience that even large retinal breaks when situated over the site of choroidal resection are not usually complicated by retinal detachment. In the case of large breaks a relatively faster clearance of subretinal fluid than its recruitment through the retinal break would seem an unlikely explanation for retinal flattening, and vitreous tamponade of the retinal hole appears a more acceptable explanation.

Whatever the explanation for retinal apposition with the scleral bed following extensive surgical resection of the choroid and RPE for choroidal malignant melanoma, it is useful that retinal detachment is a relatively uncommon complication of this surgical technique.

I am grateful to Mrs A Currie, senior technician in the Tennent Institute, for the photographs, and to Mrs J Murray for typing the manuscript.

\section{References}

1 Foulds WS. Aetiology of retinal detachment. Trans Ophthalmol Soc UK 1975; 95: 118.

2 Marmor MF, Tonnies M. Local environmental factors and retinal adhesion in the rabbit. Exp Eye Res 1982; 34: 727-33.

3 Berman ER. Mucopolysaccharides (glycosaminoglycans) of the retina-identification, distribution and possible biological role. Mod Probl Ophthalmol 1969; 8: 5-31.

4 Strambach DA, Marmor MF. The rate and route of fluid resorption from the subretinal space in the rabbit. Invest Ophthalmol Visual Sci 1982; 22: 292-302.

5 Foulds WS. The local excision of choroidal melanomata. Trans Ophthalmol Soc UK 1973; 93: 343-6.

6 Foulds WS. Experience of local excision of uveal melanomas. Trans Ophthalmol Soc UK 1977; 97: 412-5.

7 Foulds WS. Current options in the management of choroidal melanoma. Trans Ophthalmol Soc UK 1983; 103: 28-34.

8 Moseley H, Foulds WS. The movement of xenon-133 from the vitreous to the choroid. Exp Eye Res 1982; 34: 169-79.

9 Moseley H, Foulds WS, Allan D, Kyle PM. Routes of clearance of radioactive water from the rabbit vitreous. $\mathrm{BrJ}$ Ophthalmol 1984; 68: 145-51.

10 Fatt I, Shantinath K. Flow conductivity of retina and its role in retinal adhesion. Exp Eye Res 1971; 12: 218-26.

11 Moseley H. Studies of fluid movement in the eye. PhD thesis. University of Glasgow 1980: 95-6.

12 Fatt I, Hedbys BO. Flow of water in the sclera. Exp Eye Res 1970; 10: 243-9.

13 Moseley H, Johnson NF, Foulds WS. Vitreo-scleral fluid transfer in the rabbit. Acta Ophthalmol (Kbh) 1978; 56: 769-76.

14 Neji A, Marmor MF. The resorption of subretinal fluid after diffuse damage to the retinal epithelium. Invest Ophthalmol Visual Sci 1983; 24: 1475-9.

15 Foulds WS. The vitreous in retinal detachment. Trans Ophthalmol Soc UK 1975; 95: 412

16 Hammer ME. Retinal re-attachment forces created by absorption of sub-retinal fluid. In: Zauberman $\mathrm{H}$, ed. Proceedings of the Conference on Sub-retinal Space 1979. Doc Ophthalmol Proc Ser 1981; 25: 61-75. 